\title{
Gewerkschaftliche Arbeitszeitpolitik - Eine perspektivische Bilanz der ver.di Arbeitszeitinitiative
}

These 1: Die gegenwärtige gewerkschaftliche Arbeitszeitpolitik ist nicht paralysiert, sondern sie ist sehr engagiert. Leider entspricht das Ergebnis des Engagements oft nicht den (hohen) Erwartungen - vergleichbar mit dem Kampf des Don Quijote gegen die Windmühlen.

Die Schere zwischen der auf einer kritischen Analyse beruhenden Einschätzung gewerkschaftlicher Handlungsmöglichkeiten und den ungebrochen hohen Erwartungen an die gewerkschaftliche Arbeitszeitpolitik öffnet sich zunehmend. Auf der einen Seite steht das zeitlose Credo: „Entschlossene Arbeitszeitverkürzungen sind notwendiger denn je, wir brauchen endlich die 35-, besser noch die 30-Stunden-Woche"; auf der anderen Seite die nüchterne Einschätzung: „Die Gewerkschaften sind arbeitszeitpolitisch paralysiert." Dazwischen nimmt Ratlosigkeit immer größeren Raum ein. Derweil ist die offensive Arbeitszeitpolitik von der konzeptionellen Tagesordnung der Gewerkschaften verschwunden. Subversiv hat Sancho Pansa allerdings deren gut erhaltene Reste als Kuckuckseier in den Nestern modischer Themen mit öffentlicher Aufmerksamkeit und Akzeptanz (Weiterbildung, Familie, Gesundheit, Arbeitsschutz und Demografie) untergebracht. Daneben versucht Don Quijote unermüdlich, die wuchtigen Rotorblätter massiver Arbeitszeitverlängerungsforderungen mit dem Schwert zu bremsen, was ihm nur teilweise gelingt.

Die relative Erfolglosigkeit des Versuchs der Arbeitszeitbegrenzung belegen die Ergebnisse aktueller Arbeitszeitanalysen. ${ }^{1}$ Den Gewerkschaften ist es in den letzten Jahren nicht nur nicht mehr gelungen, weitere Arbeitszeitverkürzungen durchzusetzen, sie mussten ein Roll Back in der Arbeitszeitentwicklung hinnehmen. Die Arbeitszeit wurde länger, flexibler und zunehmend polarisiert. Die Ursachen sind vielfältig. Ein Grund liegt im wachsenden Umfang unbezahlter Mehrarbeit, unter anderem durch überlaufende Zeitkonten und verfallende Zeitguthaben. Ein anderer Grund ist die Nutzung tariflicher Öffnungsklauseln zur Verlängerung der Arbeitszeit. Die unter gesellschaftspolitischen und emanzipatorischen Gesichtspunkten fatalste Entwicklung ist jedoch die, dass Arbeit nach Geschlecht und Qualifikation zunehmend ungleich verteilt wird. Zugespitzt formuliert: Überlange Arbeitszeiten in (hoch) qualifizierten und gut bezahlten Tätigkeiten für Männer und Teilzeit und prekäre Beschäftigung in weniger anspruchsvollen und schlecht bezahlten Tätigkeiten für Frauen sind die zwei Seiten derselben Medaille. Frauen werden mit dieser Entwicklung immer mehr aus regulären, sozialversicherungspflichtigen Beschäftigungsverhältnissen verdrängt in solche, in denen sie mit geringen Arbeitsstundenvolumen und schlechter Entlohnung doppelt benachteiligt sind.

Hinzu kam eine aggressive und konzertierte Medienoffensive von Arbeitgebern, Politik und Teilen der Wissenschaft, die seit 2003 deutlich an Boden gewonnen hat. Die ArbeitnehmerInnen in Deutschland wurden als „Freizeitweltmeister" beschimpft. $^{2}$ Die BDA nahm besonders die - unter besonderem Verdacht der Flexibilisierungsverweigerung stehenden - ver.di-Bereiche Handel und Druck und Papier ins Visier. Die Ministerpräsidenten der Länder reihten sich in diese Phalanx ein.
Den Arbeitgebern gelang es in der Folge in verschiedenen Branchen, bestehende tarifliche Arbeitszeitstandards aufzuweichen und Arbeitszeitverlängerungen durchzusetzen:

1. Die Niederlage der IG Metall im Streik um die Angleichung der Arbeitszeit in Ostdeutschland ans Westniveau war eine wichtige Wegmarke für die Erfolge der Arbeitgeber.

2. Nach bereits bestehenden ArbeitszeitKorridor-Regelungen in der Chemieindustrie öffnete auch die IG Metall mit dem Pforzheimer Abkommen die Tür zur Arbeitszeitverlängerung auf betrieblicher Ebene zur Sicherung von Beschäftigung

\footnotetext{
1 Lehndorff, S./Wagner, A. (2004): Arbeitszeiten und Arbeitszeitregulierung in Deutschland, in: Bsirske, F. et al. (Hrsg.): Es ist Zeit: Das Logbuch für die ver.di-Arbeitszeitinitiative, Hamburg, S. 195236; Seifert, H. (2007): Arbeitszeit - Entwicklungen und Konflikte, in: Aus Politik und Zeitgeschichte 4-5, S. 17-24.

2 Wiedemuth, J. (2004): Das Wettrechnen der Milchmädchen. Arbeitszeitverlängerung als Allheilmittel gegen Wachstumsschwäche und Arbeitsmarktkrise, in: Bsirske, F. et al. (Hrsg.): Es ist Zeit: Das Logbuch für die ver.di-Arbeitszeitinitiative, Hamburg, S. 21-39.
}

Gabriele Sterkel, Dr., Tarifpolitische Grund-
satzabteilung in der ver.di-Bundesverwal-
tung, Berlin.
e-mail: gabriele.sterkel@verdi.de
Jörg Wiedemuth, Sozialwissenschaftler,
Bereichsleiter Tarifpolitische Grundsatzabtei-
lung in der ver.di-Bundesverwaltung, Berlin.
e-mail: joerg.wiedemuth@verdi.de


und Innovations- und Wettbewerbsfähigkeit der Unternehmen.

3. In der Papier, Pappe und Kunststoffverarbeitenden Industrie konnte ver.di zwar die 35-Stunden-Woche sichern; eine Öffnungsklausel ermöglicht den Betriebsparteien mit Zustimmung von ver.di jedoch die Verlängerung auf bis zu 38 Stunden ohne Lohnausgleich, im Gegenzug für Beschäftigungssicherung.

4. Im öffentlichen Dienst wurde 2006 die Arbeitszeit differenziert und für Teile der Beschäftigten verlängert. Die Arbeitgeberforderung nach der 42-Stunden-Woche konnte zwar abgewehrt werden. Der Preis dafür war jedoch eine Arbeitszeitdifferenzierung zwischen 38 und 40 Stunden für die Beschäftigten in Bund, Ländern und Gemeinden.

Aus der Perspektive von gewerkschaftlicher Programmatik und gesellschaftlichem Anspruch einer dringlich gebotenen, weiteren Arbeitszeitverkürzung erscheinen diese Zugeständnisse als Versagen der Gewerkschaften. Der Blick nach Europa dürfte dieses Urteil unterstützen. Vergleichen wir aber die tatsächlichen Zugeständnisse mit den massiven Arbeitgeberforderungen und mit der Entschlossenheit, mit der die Arbeitgeber das Projekt Arbeitszeitverlängerung - als aussichtsreichere Variante der Lohnsenkung - vorantrieben, ist das Urteil von der Niederlage zu relativieren. Im Handel und in der Druck- und Papierindustrie forderten die Arbeitgeber die Wiedereinführung der 40-Stunden-Woche. Die Arbeitgeber im öffentlichen Dienst übernahmen 2004 mit der Kündigung der Arbeitszeitbestimmungen eine Türöffnerrolle für die Arbeitszeitverlängerungspolitik der deutschen Wirtschaft ${ }^{3}$ - in der Annahme, dass hier die Durchsetzungsbedingungen am günstigsten wären.

Ver.di nahm die Herausforderung an und führte die Auseinandersetzung um die Arbeitszeit sowohl in der politischen als auch in der tarifpolitischen Arena. Im Einzelhandel zogen sich die Verhandlungen über zwölf Monate hin und waren von massiven Warnstreiks und Streiks begleitet. In der Druckindustrie dauerten die Streiks sechs Wochen, bei Papier und Pappe konnte trotz massiver Arbeitskämpfe neun Monate lang kein akzeptables Ergebnis erzielt werden. Die Versuche, in einem koordinierten Häuserkampf den Manteltarifver- trag wieder in Kraft zu setzen, waren nur zum Teil erfolgreich.

Im öffentlichen Dienst wurden mit einer Dauer von 14 Wochen die längsten Arbeitskampfauseinandersetzungen seit 80 Jahren geführt. In Krankenhäusern, Universitätskliniken und Kindergärten hatte es zum Teil nie zuvor Arbeitskämpfe gegeben. Die Premiere war kraftvoll, entschlossen und weiblich. Obwohl von vornherein klar war, dass es ein Ergebnis oberhalb der 38,5Stunden-Woche geben würde und obwohl die Angriffe in den Medien versuchten, die Legitimität der Streikenden zu untergraben, haben die Beschäftigten entschlossen gekämpft. Sie haben sich dabei auch den Auseinandersetzungen mit den BürgerInnen, die streikbedingt auf die öffentlichen Dienstleistungen verzichten mussten, gestellt - mit dem Erfolg, dass die Öffentlichkeit zum großen Teil Verständnis zeigte.

Dabei ging es den Beteiligten nicht nur um das unmittelbare Interesse an den materiellen Tarifstandards, sondern auch um die Anerkennung ihrer Arbeit und ihrer Würde als Beschäftigte. ${ }^{4}$ Die Gewerkschaften agieren zwar aus der Defensive, aber sie sind aktiv in der Defensive.

These 2: Mit der Arbeitszeitinitiative hat ver.di zwischen 2002 und 2005 den bisher letzten - groß angelegten - Versuch unternommen, aus der arbeitszeitpolitischen Defensive herauszukommen. Die Bilanz der Arbeitszeitinitiative ist ambivalent. Einerseits war die Rezeption außerhalb und innerhalb der Organisation durchweg positiv. Sie war dem Image von ver.di in der (freundlichen) Öffentlichkeit zuträglich. Andererseits war die Initiative nie wirklich in der Breite der Gliederungen von ver.di verankert und hatte nicht die angestrebten und erwarteten praktischen Konsequenzen. Ver.di ist es jedoch gelungen, dass jede Verlängerung der Arbeitszeit intern nach wie vor unter außerordentlich hohem politischen Legitimationsdruck steht.

Der Gründungskongress von ver.di hat 2001 eine arbeitszeitpolitische Initiative beschlossen, die folgende Ziele verfolgen sollte:

- die Einführung der 35-Stunden-Woche als gesellschaftliches Reformprojekt zur Beschäftigungssicherung, zur Humanisierung der Arbeitsgestaltung und als Grundlage für eine Neuverteilung der Arbeit zwischen den Geschlechtern;
- die Förderung individueller Zeitwünsche nach mehr Autonomie, Zeitsouveränität, zeitweiser Reduzierung der Arbeitszeit und zeitweiligem Ausstieg;

- die Stärkung der Schutzansprüche und Durchsetzungsmöglichkeiten der Beschäftigten und ihrer betrieblichen Interessenvertretungen.

Bereits 2002 ging es darum, Wege aus der Defensive für eine arbeitszeitpolitische Offensive zu ebnen. Die Arbeitszeitverkürzungspolitik der 1980er und frühen 1990er Jahre stagnierte seit einigen Jahren, die Unternehmen trieben vor allem mit Arbeitszeitkonten die Flexibilisierung der Arbeitszeit voran, die tatsächlichen Arbeitszeiten lösten sich schleichend und zunächst wenig offensichtlich von den tariflichen und wurden länger. Die Unternehmen beschworen die Win-Win-Situation: Beide Seiten sollten von neuen Arbeitszeitarrangements profitieren. Eine effektivere Arbeitsorganisation mit flexiblen Arbeitszeiten sollte gleichzeitig mehr „Zeitsouveränität“ für die Beschäftigten mit sich bringen. Eventuelle negative Folgen wie überlaufende Zeitkonten, verfallende Zeitguthaben und zunehmender Leistungsdruck erschienen eher als unglücklicher Nebeneffekt einer an sich für beide Seiten vorteilhaften Entwicklung, dem mit regulierenden Vereinbarungen beizukommen wäre.

Die Arbeitszeitinitiative hatte in verschiedener Hinsicht einen für gewerkschaftliche Kampagnen völlig neuartigen und ungewöhnlichen Ansatz. Sie war beteiligungs- und prozessorientiert. Sie bezog von vornherein WissenschaftlerInnen und die interessierte Öffentlichkeit mit ein; die neue Ausrichtung der Arbeitszeitpolitik von ver.di sollte auf Grundlage aktueller sozialwissenschaftlicher Erkenntnisse stattfinden. ${ }^{5}$

3 Bsirske, F. (2005): Arbeitszeitverlängerung in Zeiten der Massenarbeitslosigkeit: ein Projekt der gesellschaftlichen Gegenreform, in: Bsirske, F. et al. (Hrsg.): Perspektive neue Zeitverteilung. Logbuch 2 der ver.di-Arbeitszeitinitiative: für eine gerechte Verteilung von Arbeit, Zeit und Chancen, Hamburg, S.13-23.

4 Stamm, S./Busch, G. (2006): Politisch und moralisch gewonnen - materiell eine halbe Stunde verloren. Der Streik der Kommunalbeschäftigten in Baden-Württemberg, in: Sozialismus 5, S. 39-44.

5 Sterkel, G. (2004): Arbeitszeitinitiative auf schwierigem Terrain, in: Bsirske, F. et al. (Hrsg.): Es ist Zeit: Das Logbuch für die ver.di-Arbeitszeitinitiative, Hamburg, S. 9-20. 
Die verschiedenen Branchen und Ebenen von ver.di sollten sich über gemeinsame Ziele und Schritte in der Politik der Arbeitszeitverkürzung und über die notwendige Regulierung der Flexibilisierung verständigen. Eine Kampagne nach dem in Gewerkschaften üblichen Top-DownMuster galt bei der Heterogenität der Beteiligten und der Handlungsautonomie der verschiedenen Organisationsteile von vornherein als wenig aussichtsreich. Die Initiative wurde also als mehrjähriger Prozess der Verständigung angelegt, an dessen Ende nicht nur eine gemeinsame arbeitszeitpolitische Ausrichtung der Organisation, sondern schließlich eine gemeinsame Tarifbewegung stehen sollte.

Die vielfältigen Arbeitszeitprobleme, -praxen und -konzepte der unterschiedlichen Branchen in ver.di wurden analysiert, Unterschiede identifiziert und branchenübergreifende, verallgemeinerungsfähige Ansatzpunkte für die gemeinsame Initiative herausgearbeitet. Der zweite neue wesentliche Ansatzpunkt lag in der Akzep$\operatorname{tanz}$ der Unterschiedlichkeit der realen Arbeitszeitwünsche und -interessen einzelner Beschäftigtengruppen. Für die Beschäftigten wurden Aktionsmedien wie der „Arbeitszeitkalender“ und das "Stressbarometer" entwickelt, die Reflexion über das Bewusstsein der eigenen Arbeitszeitsituation bei den Beschäftigten befördern sollten, die dann in Betrieb und Verwaltung diskutiert, zu kollektiver Reflexion und zur Bereitschaft zur aktiven Veränderung der Arbeitszeitverhältnisse führen sollten. Das aufwendigste Beteiligungsprojekt war die Arbeitszeitumfrage: eine groß angelegte Mitgliederbefragung mit umfangreichem Fragebogen in der Mitgliederzeitung PUBLIK und im Internet, an der sich immerhin 25.000 Mitglieder und Nichtmitglieder beteiligt haben. ${ }^{6}$

Die Abwehr von Arbeitszeitverlängerung wurde bald zum unvorhergesehenen Schwerpunkt der Initiative. Mit der „Perspektive neue Zeitverteilung" wurde versucht, der zunehmenden Leistungsintensivierung, gesundheitlichen Risiken und der wachsenden Ungleichverteilung von Arbeit(szeit) nach Geschlecht und Qualifikation offensiv mit der Idee einer „biografieorientierten Arbeitszeit" ${ }^{\text {"7 }}$ zu begegnen.

These 3: Die Ursachen für die mangelhafte Durchsetzungsfähigkeit einer offensiven gewerkschaftlichen Arbeitszeitpolitik zur arbeitsmarktpolitisch und geschlechterdemo- kratisch wirksamen Arbeitszeitverkürzung und zur wirksamen Regulierung der Arbeitszeitflexibilisierung im ArbeitnehmerInneninteresse sind vielfältig. Sie sind auch in den objektiven und ideologischen Bedingungen einer Lohnkostensenkungs- und Wettbewerbshysterie zu finden, die mit Massenarbeitslosigkeit und Absturzbedrohung Angst, mit Reallohnsenkungen, dem Ausbau des Niedriglohnsektors, zunehmender Prekarisierung und abnehmender sozialer Sicherung materielle Not erzeugt - und damit bei ArbeitnehmerInnen eine Präferenz für mehr Geld statt mehr Freizeit auslöst.

Die erfolgreiche Arbeitszeitverkürzungspolitik der 1980er Jahre wurzelte unter anderem im postmateriellen Wertesystem der Wohlstandsgesellschaft am Ende einer langen Prosperitätsphase. Die neuen sozialen Bewegungen wie Ökologie-, Alternativ-, Friedens-, Frauenbewegung waren Ausdruck dieses Wertewandels. Die Forderung nach der 35-Stunden-Woche konnte an den Vorstellungen von einem nachhaltigeren, auf mehr Lebensqualität gerichteten Wirtschaften ansetzen. Arbeitsmarktpolitische Zielsetzungen von Arbeitsumverteilung verbanden sich mit einer Vision vom Ende der Zentralität der Arbeit und einer geschlechtergerechteren Gesellschaft. Heute sind - nach 20 Jahren Reallohnverzicht, Massenarbeitslosigkeit, Sozialstaatsabbau, wachsendem Niedriglohnsektor, zunehmender Prekarität und Armut - postmaterielle Werte ins Hintertreffen geraten. Arbeitszeitverlängerung ohne Lohnausgleich ist nun die Variante des Lohnverzichts, die von vielen Beschäftigten noch am besten verkraftet werden kann. Der jahrzehntelange Kaufkraftverlust hat die ökonomischen Spielräume der Beschäftigten, die es ermöglichen könnten, einen Teil des Verteilungsspielraums für Arbeitszeitverkürzung zur Verfügung zu stellen, reduziert.

Angesichts von Massenarbeitslosigkeit und der Zuspitzung sozialer Unsicherheit und Abstiegsbedrohung durch Hartz IV hat sich eine hohe Anpassungsbereitschaft entwickelt. Quasi über Nacht kann man durch Arbeitsplatzverlust aus der Mittelschicht ins soziale Abseits katapultiert werden, ohne Auffangnetz und ohne Wiedereinstiegsmöglichkeiten - zumal ab einem bestimmten Alter. Angst vor Arbeitsplatzverlust lässt allzu oft nach individuell-konkurrenzorientierten statt solidarischen Lösungen suchen. Offensichtlich scheint es vielen plausibler, auf die existenzielle Be- drohung durch Arbeitslosigkeit nicht mit einer kollektiven Verknappung des Arbeitskraftangebots durch Arbeitszeitverkürzung $\mathrm{zu}$ reagieren, sondern mit einem individuellen Zusatzangebot an Arbeitsvolumen, um die persönliche Leistungsbereitschaft als Wettbewerbsvorteil zu demonstrieren. Verstärkt wird der Trend zur Entsolidarisierung noch durch die Prozesse der Vermarktlichung und Ergebnisorientierung, mit denen den Beschäftigten die Verantwortung für den Unternehmenserfolg aufgeladen wird.

Beim gewerkschaftlichen Subjekt ist nicht selten ein gespaltenes Bewusstsein festzustellen. Einerseits ist es meist durchaus in der Lage, allgemein Ökonomisierung der Zeit und Vermarktlichung als Herrschaftsverhältnisse zu identifizieren und deren Alternativlosigkeit infrage zu stellen, andererseits hat es sich im konkreten und unmittelbaren Arbeitszusammenhang die betriebswirtschaftliche Rationalität tief ins Bewusstsein eingraben lassen. Wenn im Betrieb mit Lohnverzicht und Arbeitszeitverlängerung Arbeitsplätze gerettet werden können, dann sind die gesamtwirtschaftlichen und arbeitsmarktpolitischen Folgen meist sehr weit weg.

Anders als in den 1980er Jahren ist es den Gewerkschaften bei ihren jüngeren Kämpfen um die Zeit sehr unzureichend gelungen, soziale Bündnisse zu schmieden. Die Gewerkschaften können in der Öffentlichkeit kaum noch vermitteln, dass eine Politik der Arbeitszeitverkürzung Arbeitsplätze schafft und in gesamtgesellschaftlichem Interesse ist. Der Streik der IG Metall in Ostdeutschland wurde vor allem aus diesem Grund verloren. Es wurde nicht genügend um die Unterstützung der Bevölkerung gekämpft, die, indoktriniert von der

6 Lehndorff, S. (2005): Herausforderungen an die Arbeitszeitpolitik der Gewerkschaften. Arbeitszeitprobleme und -wünsche von Dienstleistungsbeschäftigten im Spiegel der ver.di-Arbeitszeitumfrage, in: Bsirske, F. et al. (Hrsg.): Perspektive neue Zeitverteilung. Logbuch 2 der ver.di-Arbeitszeitinitiative: für eine gerechte Verteilung von Arbeit, Zeit und Chancen, Hamburg, S. 56-71.

7 Mönig-Raane, M. (2004): Wem gehört die Zeit? Koordinaten einer anderen Zeitverteilung, in: Bsirske, F. et al. (Hrsg.): Es ist Zeit: Das Logbuch für die ver.di-Arbeitszeitinitiative, Hamburg, S. 255267; vgl. außerdem die Beiträge in: Bsirske, F. et al. (Hrsg.) (2005): Perspektive neue Zeitverteilung. Logbuch 2 der ver.di-Arbeitszeitinitiative: für eine gerechte Verteilung von Arbeit, Zeit und Chancen, Hamburg. 
neoliberalen Meinungsmache, mehrheitlich den Streik nicht als Chance für mehr Beschäftigung und Angleichung der Lebensverhältnisse in Ost und West begriff. Ein ähnliches Problem hatte auch ver.di beim Streik im öffentlichen Dienst 2006. Dass die Streikenden im allgemeinen gesellschaftlichen Interesse handelten, indem sie den Abbau von Arbeitsplätzen durch Arbeitszeitverlängerung versuchten zu verhindern, konnte nicht wirklich vermittelt werden.

These 4: Chancen für eine neue gewerkschaftliche Arbeitszeitpolitik eröffnen sich da, wo es gelingt, die Forderung nach Arbeitszeitverkürzung in den Kontext von erfahrbarem größerem individuellen Zeitwohlstand $z u$ stellen. Sie eröffnen sich da, wo sie mit dem gesellschaftlichen Interesse an nachhaltiger - auf den Erhalt von Gesundheit und Qualifikation orientierter - Arbeit verbunden wird, wo sie am Bedürfnis der Beschäftigten nach einem Gleichgewicht von Arbeit und Leben, nach der Vereinbarkeit von Beruf und privaten Erfordernissen und nach einer gerechteren, egalitäreren Verteilung von Arbeit, Zeit und Lebens- und Erwerbs-Chancen ansetzen kann.

Die strategische Verknüpfung von Themen wie Gesundheit, Qualifikation, Balance von Arbeit und Leben mit der Arbeitszeitpolitik verlangt eine Antwort auf die Frage: „Was ist eine gute Arbeitszeit?“. Dabei wäre es wenig hilfreich, den Herrschaftsaspekt der Zeit auszublenden. Die Frage nach der Fremd- oder Selbstbestimmung der Zeitverwendung („Wer verfügt über unsere Lebenszeit?") spielt dabei eine ebenso zentrale Rolle wie der Aspekt der Gesellschaftlichkeit der Zeit („Zeit ist nicht gleich Zeit"). Der allseitigen Verwandlung der Zeit in Arbeitszeit oder Konsumzeit müssen wir ein von Wertüberzeugungen getragenes Konzept der gesellschaftlich geschützten Zeit gegenüberstellen. Die Qualität kollektiver Zeitrhythmen kann nicht durch das Zeitmanagement individueller Freizeitpuzzles aufgewogen werden.

Kann die Wiederbelebung der Arbeitszeitpolitik dadurch gelingen, dass wir sie in die aktuellen öffentlichen und wissenschaftlichen Diskurse - wie z. B. Familie, Bildung und Demografie - integrieren? Oft wird eine gewisse Interessenübereinstimmung mit der Arbeitgeberseite in diesen Fragen beschworen. Zwar häufen sich neuerdings Hinweise und Untersuchun- gen, die der Entwicklung der einzelnen Aspekte des „Humankapitals“ unter Nachhaltigkeitsgesichtspunkten und langfristigen ökonomischen Wirkungen für die Wohlfahrt der Volkswirtschaft einen höheren Stellenwert zuschreiben. Ob dies jedoch ausreicht, um die Blockade der Unternehmen und Arbeitgeberverbände aufzulösen, ist nicht von vornherein mit „Ja“ zu beantworten.

Die Beschäftigten - das haben die Erfahrungen des Arbeitskampfs im öffentlichen Dienst, und insbesondere im Krankenhausbereich gezeigt - sind bereit, sich gegen die Verschlechterung ihrer Arbeitsbedingungen einzusetzen. Ein Plus an Legitimität und Durchsetzungsfähigkeit gewinnt der Streit um die Zeit da, wo z. B. das Interesse der Beschäftigten an kürzeren Arbeitszeiten mit dem Interesse der Öffentlichkeit und der PatientInnen an einer besseren Pflege übereinstimmt. Das belegen die Streiks in den Krankenhäusern in Baden-Württemberg, aber auch die Kampagne der Ärzte im Marburger Bund.

Die Voraussetzung für das Gelingen der „integrierten Arbeitszeitpolitik“ ist also die Identifikation der Beschäftigten mit einer nachhaltigen - auf den Erhalt von Gesundheit, Qualifikation, Balance und Chancengleichheit orientierten Zielsetzung, die ihnen die nötigen Spielräume für unterschiedliche Lebens- und Karriereentwürfe lässt. Sie muss von ihnen selbst als existenziell wahrgenommen und auch politisch vertreten werden. Ein derartiges Gesundheits- und Arbeitszeitbewusstsein ist aber erst zu fördern, ehe es zum Träger eines neuen arbeitszeitpolitischen Aufbruchs werden kann.

Die ver.di Arbeitszeitinitiative muss daher noch längerfristiger gedacht werden. Wesentlicher Bestandteil muss sein, Bewusstseinsbildung mit konkreten Aktivitäten zu verbinden, die auch den Erhalt des Status quo und die Abwehr von Angriffen der Arbeitgeberseite zum Ziel haben kann. Insofern birgt jede Arbeitszeitauseinandersetzung die Chance in sich, aus der Defensive heraus Bedingungen für eine Offensive $\mathrm{zu}$ entwickeln. Politische Auseinandersetzungen kann man sich nicht immer aussuchen. In mehreren ver.di Tarifbereichen (z. B. im öffentlichen Dienst) werden die Arbeitgeber eine weitere Verlängerung der Arbeitszeit verlangen. Dieser Herausforderung gilt es offensiv zu begegnen. Der wichtige Schritt, die Arbeitszeitverlängerung zu verhindern, ist dabei nicht einfach zu über- springen und eine weitere Arbeitzeitverkürzung zu fordern.

Diskussionen um alternde Belegschaften, die Erhöhung des Rentenalters und die Vereinbarkeit von Familie und Beruf für Frauen und Männer bieten die Chance, für längerfristige Konzepte zu werben. Verkürzung der Arbeitszeit für Ältere ist da sinnvoll, wo die Beschäftigten starken Belastungen ausgesetzt sind. Viel sinnvoller ist es, die Arbeit(szeit) über den Erwerbsverlauf, in der Erwerbsbevölkerung, zwischen den Geschlechtern gerechter zu verteilen. Ver.di setzt sich deshalb ein für eine biografieorientierte Arbeitszeit, die eine Kumulation von beruflichen und privaten Anforderungen in bestimmten Phasen des Lebens entzerren hilft und längere Phasen der Freistellung im Erwerbsverlauf ermöglicht. ${ }^{8}$ Damit soll eine bessere Balance erreicht und der zunehmenden Polarisierung Einhalt geboten werden: durch die Umverteilung der Arbeit(szeiten) zugunsten der "ArbeitszeitverlängerungsverliererInnen“ - der Frauen, der Erwerbslosen, aber auch der gestressten Vielarbeiter.

Ohne gesellschaftliche Bündnispartner und die Vision einer gerechteren Gesellschaft wird da kein Durchbruch zu erzielen sein. Deshalb ist eine Politisierung gewerkschaftlicher Arbeitszeitpolitik unabdingbar:

- Im Zusammenhang mit der Demografiediskussion lässt sich der Zusammenhang zwischen Arbeitszeitdauer, Arbeitszeitverteilung und daraus erwachsenden Belastungswirkungen diskutieren. Deutlich werden muss dabei auch, dass ohne ein neues Zeit- und Gesundheitsbewusstsein ein Arbeitsleben bis zur Rente immer unwahrscheinlicher wird.

- Die herkömmliche Rollen- und Arbeitsverteilung zwischen den Geschlechtern wird den veränderten Ansprüchen der Menschen nicht mehr gerecht. Zudem werden konservative Rollenmuster immer stärker zu Entwicklungs- und Produktivitätsbremsen angesichts sektoraler Arbeitskräfteangebotsungleichgewichte.

\footnotetext{
Barkholdt, C. (2005): Arbeits(zeit)verteilung in der Lebenslaufperspektive, in: Frank Bsirske et al. (Hrsg.): Perspektive neue Zeitverteilung. Logbuch 2 der ver.di-Arbeitszeitinitiative: für eine gerechte Verteilung von Arbeit, Zeit und Chancen, Hamburg, S. 121-129.
} 
Die modernisierende Wirkung auf Gesellschaft, Wirtschaft und Arbeitskräftepotenzial erschließt sich jedoch nur in der gesamtgesellschaftlichen Perspektive und nicht aus der Sicht von kurzfristigen, an Börsenkursen orientierten Einzelkapitalinteressen. Dem staatlichen Beschäftigungssektor und einem Dienstleistungsbereich, der auf gesamtgesellschaftlich vermittelte Dienstleistungen orientiert ist, könnte bei einem derartigen „Dienstleistungsverständnis" eine Schlüsselrolle bei der sozialen Modernisierung der Gesellschaft zukommen.
Der aktuelle Konjunkturaufschwung stellt unsere arbeitszeitpolitischen Überlegungen auf ein neues, realistisches Fundament. Es kommt zunächst darauf an, dass die Segnungen des Wachstums auch bei der breiten Mehrheit der Beschäftigten ankommen, was keineswegs selbstverständlich ist. Aktive Lohnauseinandersetzungen sind ein wichtiger Schritt aus der gewerkschaftlichen Defensive. Sie bringen Entspannung im Budget der ArbeitnehmerInnen und auf dem Arbeitsmarkt und sie steigern das Selbstbewusstsein. Die weitverbreitete Angststarre und Anpassungs- neigung vieler Belegschaften kann so Schritt für Schritt aufgelöst werden. Die vielfältigen Initiativen zur Verbesserung von Arbeitsbedingungen und -zeiten, zur Bekämpfung von Stress und Leistungsdruck, für mehr Gesundheit und Weiterbildung, die es bei ver.di gibt, haben endlich eine ernsthafte Chance, ihre lebensfreundliche und emanzipatorische Wirkung zu entfalten.

\title{
"Von Finnland lernen?"
}

\author{
Gemeinsamer Workshop des Wirtschafts- und Sozialwissenschaftlichen Instituts (WSI) \\ in der Hans-Böckler-Stiftung und der IG Metall
}

\section{Juni 2007 in Frankfurt/Main}

Finnland hat es in den vergangenen Jahren geschafft, in die Reihe der führenden Industrienationen aufzusteigen. Es gilt heute als Modell einer sich rasch entwickelnden Wissensgesellschaft, in der es gelingt, hohe Wachstumsraten mit einem funktionierenden Sozialstaat zu verbinden. Was können wir von den skandinavischen Erfahrungen lernen? Welche Bedingungen haben den Strukturwandel ermöglicht? Wie lässt sich das finnische Modell der Wissensökonomie charakterisieren? Welche Rolle können Betriebsräte und Gewerkschaften in solchen Wandlungsprozessen spielen?

Diese und andere Fragen sollen im Workshop erörtert werden. Folgende Referate sind u. a. vorgesehen:

- Die finnische Forschungs-, Technologie- und Innovationspolitik - Entwicklungen und Perspektiven (Prof. Dr. Gerd Schienstock)

- Skandinavien und Deutschland im Innovationswettbewerb - Ein Vergleich aus Sicht der Wirtschaftsforschung (Dr. Harald Legler)

- Innovationspolitik: Wie kann Deutschland von anderen lernen? (Dr. Astrid Ziegler)

- Von Nixdorf über ... BenQ zu möglichen Antworten der IG Metall auf strukturelle Herausforderungen (Franz Tölle)

Anmeldung und Information: Heike Stempel, Hans-Böckler-Stiftung; e-mail: heike-stempel@boeckler.de 